\title{
ZUR GESCHICHTE UND GEOMORPHOLOGIE DES NORDSCHWEIZERISCHEN JURAGEBIRGES
}

\section{Hans Liniger}

\section{Mit 3 Abbildungen}

Nachdem der Basler und Berner Jura lückenlos durch geologische Aufnahmen 1:25000 von Basel bis in die Freiberge erforscht wurden, nachdem durch zwei große Tunneldurchstiche Licht in den innern Aufbau der großen Ketten fiel 12, nachdem durch mehrere neue Arbeiten auch die morphologischen Verhältnisse weitgehend abgeklärt werden konnten ${ }^{\mathbf{4 9}},{ }^{\mathbf{4 2}}, \mathbf{2 0}$, ist der Zeitpunkt für eine Synthese gekommen. Zivei morphologische Probleme sind noch umstritten: die Entstehung der imposanten Faltenquertäler (Klusen), sowie Bildung und Datierung der Einebnungsfläche an der Wende des Miozäns zum Pliozän. Da beide Fragen in enger Beziehung zum Gang der Auffaltung stehen, so soll im folgenden die Tertiärsedimentation genau untersucht werden, wobei ich mich auf das Miozän beschränke; die Einbeziehung des Oligocäns würde den Rahmen der Arbeit sprengen.

Vorerst sei das Werden der Landschaft seit der Eozänzeit kurz umrissen, wobei auf ähnliche Darstellungen wie diejenige von VosseLER ${ }^{j 1}$ verwiesen sei. Seit dem Zurückweichen des Kreidemeeres aus dem $s$ Juragebiet bestand in Mitteleuropa ein Festland, dessen Verwitterungsprodukte uns in den eozänen Hupper-Quarzsanden, den Roterden (Bolus) mit Eisenkonkretionen (Bohnerz), Süßwasserkalken und eozänen Konglomeraten erhalten geblieben sind. Es entwickelte sich eine Fastebene, die vom Schweizer Mittelland gegen den Schwarzwald-Vogesenhorst schwach anstieg. In der nachfolgenden Oligozänzeit zerbrach die alte Fastebene, soda $\beta$ durch den neu gebildeten Rheintalgraben das Meer von $\mathrm{N}$ her eindringen konnte und in einer breiten Senke, die quer durch den Jura verlief - Raurachische Senke nach BAUMBERGER ${ }^{6}$ - seine Spuren hinterließ, w der großen Rheintalflexur, die von Basel südwärts noch bis Äsch erkennbar ist (Fig. 1). Die marine und brackische Molasse süßte sich im Oberstampien aus und es kam zur Bildung eines jurassischen Süßwasserkalks, des Delsbergerkalks, auch Delémontien genannt, der eine Sedimentationsphase abschloß. Im Früholigozän war der Rheintalgraben eingebrochen, wobei sich Begleitbrüche aller Art bildeten, die weit nach $S$ in das Gebiet des spätern Kettenjuras reichten (Fig. 1); vor Mitteloligozän war das Gebiet der Ajoie zu einer oligozänen Fastebene gewandelt worden. Ablagerungen des Oberoligozäns erscheinen nur am Jurasüdfuß und zwar in großer Mächtigkeit; es bestand also in der Aquitanzeit im Jurainnern eine Sedimentationslücke.

Die neu heranrückenden Miozänmeere hinterließen im Burdigalien in den südlichsten Juramulden Molasse grise und Muschelsandstein, im Helvetien eine $n$ Strandlinie (Tenniken-Ziefen-MettembergMontfaucon) mit Muschelbrekzien auf Malm und eine südliche Nagelfluhlinie auf Tertiär. Sie ist als bunte Nagelfluh oder poudingue polygénique bekannt und stammt zum Teil von $N$, z. T. von den Alpen. Ebenfalls von $\mathrm{N}$ müssen die Juranagelfluh und die Dinotheriensande, beides tortonische Bildungen kommen, wodurch eine Heraushebung der Vogesen-Schwarzwald-Horste dokumentiert wird. Im Tafeljura ist die Juranagelfluh vergesellschaftet mit roten Landschneckenmergeln mit Helix silvana, einem Aequivalent der Silvanahorizonte Südschwabens. Im W stellt BAUMBERGER ${ }^{23}$ die Süßwasserkalke mit Melania Escheri (Brotia Escheri) in denselben Horizont, obschon Wesz dieses Niveau im Mainzerbecken dem jüngern Sarmatien zuzählt ${ }^{53}$. Im Bernerjura schließen sich die Melanienkalke und die tortonischen Flußbildungen aus, was für Gleichaltrigkeit sprechen könnte.

Typisch jünger sind die Vogesenschotter von Charmoille und des Bois de Raube bei Delsberg, seitdem Hummer. ${ }^{27}$ von Charmoille eine Pontienfauna mit Hipparion gracile Kaup und Dinotherium giganteum Kaup beibringen konnte. Diese für die Jurafragen ausschlaggebende Datierung scheint neuerdings ins Schwanken zu geraten, seit TOBIEN ${ }^{4 \overline{5}}$ in den sarmatischen Öhningerschichten des Hegau Hipparion gracile feststellte. Nun liegen aber im Mainzerbecken und in der Wetterau die Dinotheriensande mit der Unterpliozänfauna von Eppelsheim über den Congerienschichten, die im Wienerbecken ebenfalls zum Pontien (Pannon) gestellt werden ${ }^{40}$. SCHAFFer zitiert Hipparionfunde aus dem untern und obern Pannon, wodurch sich auch dort zwei Horizonte des Pannon (=-Congerienschichten) mit Hipparion ergeben. Bis zur Revision der Gattung Hipparion, die das Leitfossil des europäischen Unterpliozäns ist, und der damit verbundenen Gesamtrevision der Grenze des Sarmat und des Pontien (Unterpliozän) lasse ich die Charmoillekiese beim Pontien und rechne die ebenfalls Hipparion - führenden Hegautuffe zum Sarmatien, obschon auch Buxrorf die Möglichkeit der Verlegung der Charmoilleschotter in eine etwas ältere Stufe in Betracht zieht ${ }^{13}$.

Ungeklärt ist die Altersfrage der Quarzitschotterrelikte, die man vielfach auf den Höhen des Juras trifft. Im W sind sie unter dem Namen Höhenschotter beschrieben; im Laufenbecken hat KocH sie Wanderblockformation genannt. RU'TrE ${ }^{38}$ hat letztere der Juranagelfluh und damit dem Torton zugewiesen; nachdem nun aber Witrmans ${ }^{54}$ die Heubergschotter bei Kandern dem Pontien 


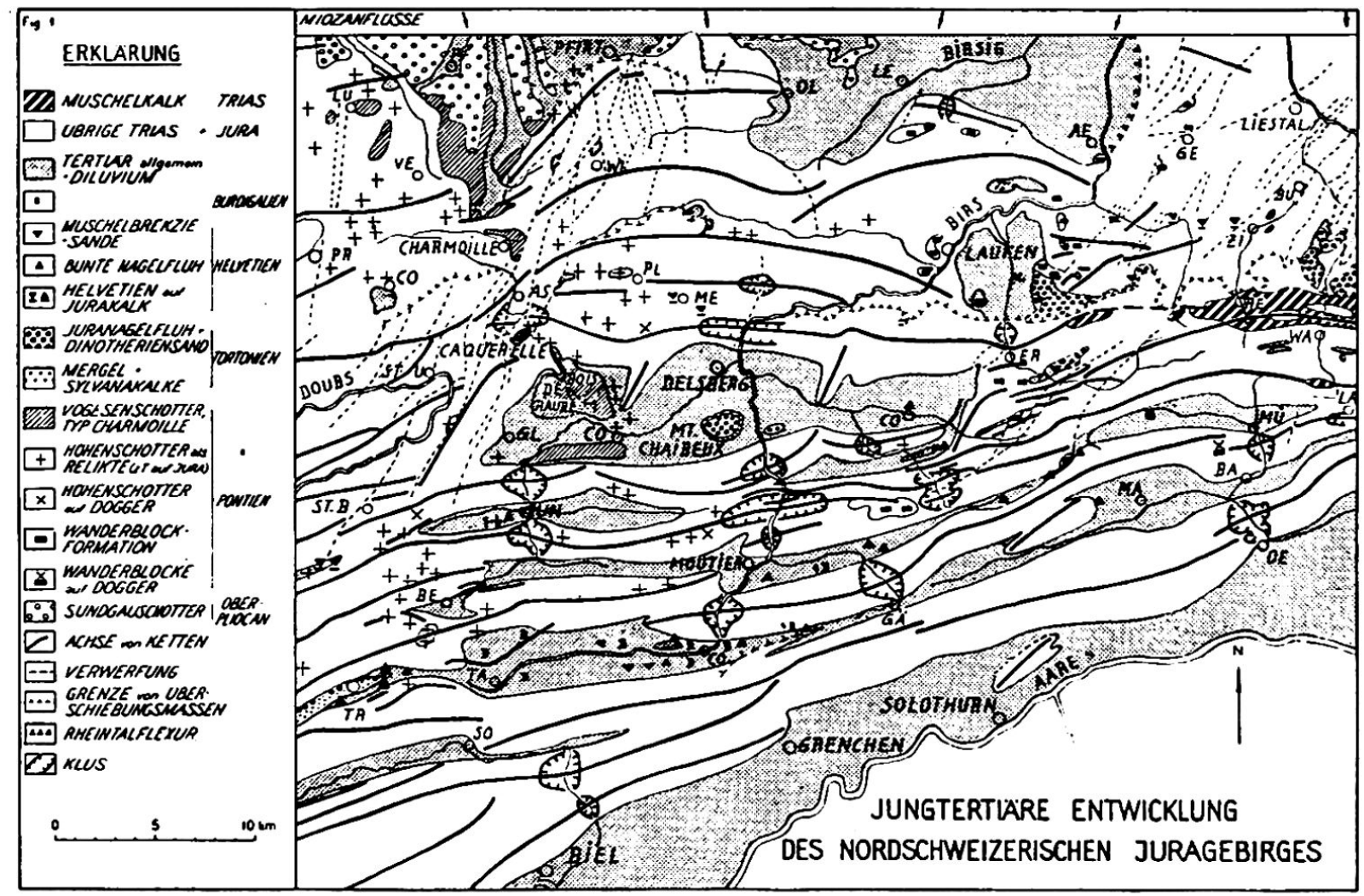

gleichstellt, ist die Wahrscheinlichkeit wieder größer, daß sie doch zum Unterpliozän gehören. WitrmanN hat übrigens bei Lörrach n Basel tortonische Konglomerate glaubhaft gemaclit; sie gleichen der Wanderblockformation keineswegs.

Folgende Gründe bewegen mich, die umstrittenen Höhenschotter, die für die Datierung der Faltungsphasen des Gebirges wichtig sind, den pontischen Charmoillesanden gleichzustellen: Sie kommen auch da vor, wo weder Juranagelfluh noch Helvetien abgelagert wurden; sie begleiten randlich die Charmoillekiese ${ }^{20}$, was ERzINGER in der Ajoie überzeugend nachwies, sie zeigen die typischen Schlagfiguren wie die Vogesenschotter, sie haben überall Verkieselungserscheinungen, Bleichungen und sind mit Brauneisenkonkretionen behaftet und vergesellschaftet, wie übrigens die Wanderblöcke auch. Erst Fossilfunde könnten die Altersfrage dieser Quarzschotterrelikte definitiv lösen.

\section{Fine neue Theorie der Klusenentstehung.}

Eine eingehende Darstellung der Juraklusformen, ihrer Entstehungtheorien und ihrer Literatur findet sich in Herms Geologie der Schweiz, Band I ${ }^{26}$. ThURıAN führte den Namen Kluse (mittellateinisch clusa $=$ Engpaß) ein, woran WYss erinnerte ${ }^{j \tilde{a}}$. SiUdER uud GressLY erklärten ihre Entstehung durch vulkanische Risse; später wurden rein erosive Vorgänge wie Rückwärtserosion oder tektonische Bedingtheiten der Kettendurchbrüche in Betracht gezogen. Gewiß gibt es auch Klusen, die an bedeutenden Querstörungen entstanden, wie im Waadtländerjura. A. HeIM stellt sich auf die Seite derjenigen, die z. B. in den bekannten Birsklusen, die mehrere Falten direkt queren, ein antezedentes Stromsystem erblickten; er faßt die Urbirs auf als entstanden nach der Jurafaltung im Pliozän, die von der ersten (und höchsten) Jurakette im S nach $N$ abgeflossen sei. ELbf: ${ }^{19}$, der letzte Bearbeiter der Juraklusen bei Moutier, meinte, daß eher ein Fluß aus dem $\mathbf{N}$ die Klusen geschaffen hätte, wobei er zwei Jurafaltungen voraussetzte. Neuerdings dachte R. STAuB auch an alpine Flüsse, die das Mittelland und das Gebiet des spätern Jura gequert hätten, die für die Klusenanlage verantwortlich sein könnten ${ }^{4+*}$

Im folgenden sei nun versucht, die Frage durch das Studiun der Tertiärschichten und der tertiären Erosionen zu lösen.

Begeben wir uns zuerst auf den Hügel Mont Chaibeux s Delsberg. Dort fehlt die auffallende Tafel der stampischen Delsbergerkalke (Fig. 2). Sie ist aber weder durch diluviale noch durch helvetische Flußerosion entfernt worden, denn in den Basislagen des Hügels trifft man Gerölle von angebohrtem Delsbergerkalk und umgelagerte Helvetienfossilien; zudem ruht das Helvetien bei Corban auf Oligozän auf ${ }^{52}$. Da der ganze Komplex des Mont-Chaibeux mit seinen Vogesensanden, die

* Neuestens haben sich auch ViRieux ${ }^{* 0}$ und KuTrer (Klusen und Höhlen. St. Margrethen 1950) mit der Klusenentstehung befaßt. 
Dinotherium Bavaricum führen, zum Torton zu stellen ist, so muß der Fluß aus dem $\mathrm{N}$, der die Rinne ins Oligozän eingeschnitten hat, vortortonisch erodiert haben. Die Breite dieses tertiären Tales betrug ca $4 \mathrm{~km}$ (Fig. 2); es hatte Talränder von $80 \mathrm{~m}$ Höhe. Es liegt deshalb eine Rinne von beachtlichem Ausmaß vor, die natürlich auch Fortsetzungen nach $\mathrm{N}$ und $\mathrm{S}$ gehabt hatte, obschon bei Delsberg die Rinne aufgefüllt wurde und weiter s keine Vogesensande festzustellen sind. Dagegen fällt nun sofort auf, daß in der s Fortsetzung der Chaibeuxrinne die Klusen von Moutier und in der $\mathrm{n}$ Fortsetzung diejenigen von Soyhières liegen. Daraus möchte ich den Schluß ziehen, daß damit ein erster Hinweis einer Urbirs aus dem $\mathrm{N}$ vorliegt, womit die 'Theorie Elbers Gestalt annimmt.

Fig. 2

\section{MIOCANE FLUSSRINNEN BEI DELSBERG}
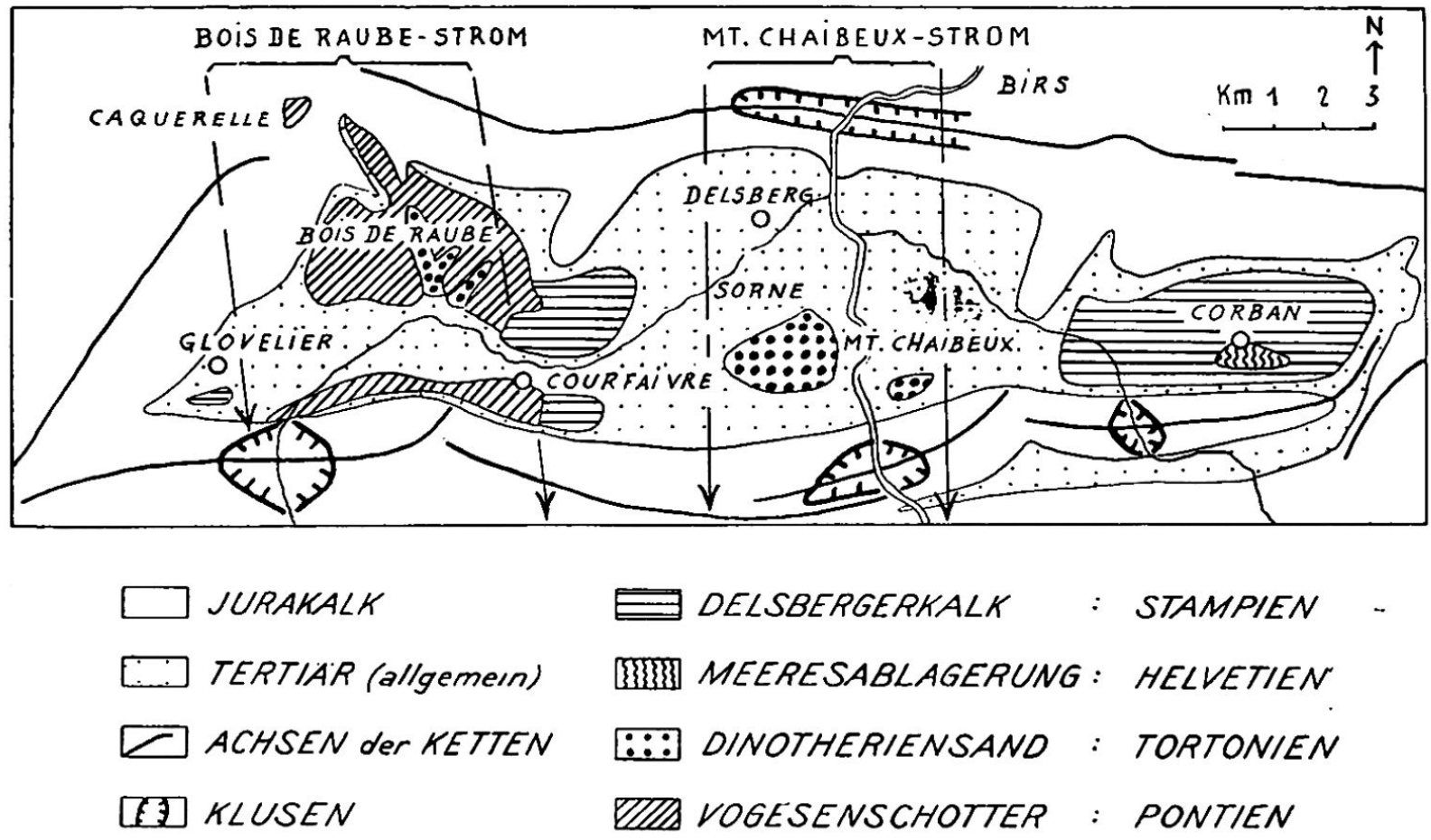

Im. Delsbergertal findet sich nun noch eine zweite, ähnlich beschaffene Rinne; die Lücke der Delémontientafel zwischen Courfaivre und Glovelier ist auch mit jungtertiären Vogesensanden und zwar mit denen des Bois de Raube ausgefüllt (Fig. 2). Nur liegt dort insofern eine Komplikation vor, als über einem teilweise entfernten Torton als Rinnentiefstes der jüngere Schotter des Pontien ruht; der pontische Fluß muß also das ältere Tortontal weiterbenützt haben Diese w Rinne ist von ähnlichem Ausmaß wie diejenige unter dem Chaibeux und quert die heutige Mulde ebenfalls von $\mathrm{N}$ nach $\mathrm{S}$. Nach $\mathrm{N}$ hängen die pontischen Bois de Raubeschotter augenfällig mit den Höhenschottern der Caquerelle zusammen; in der Ajoie sind sie bei Charmoille und Lugnez bekannt, wo sie Hipparion führen 20. Damit ist die Stromrichtung solcher Vogesenflüsse eindeutig festgelegt. Daß dieser jungtertiäre Fluß eine bereits bestehende Rangierskette gequert hat (bei der Caquerelle), läßt sich dadurch nachweisen, daß in den Schottern der Ajoie bei Charmoille Gerölle von Jurakalk sozusagen völlig fehlen, während sie im Bois de Raube weiter s in gröBerer Zahl vorkommen 32. Damit ist die Paßlücke der Caquerelle mit ihren Höhenschottern als tertiäre Stromrinne, als uralte Klusenanlage gekennzeichnet. Daß sie später - nebenbei gesagt - nicht wie andere Querrinnen zum großen Klusenzirkus eingetieft wurde, ist wohl darauf zurückzuführen, daß dort später eine vorerst nebensächliche $W$ asserscheide entstand. Mit diesen Ausführungen wäre also 
der direkte Nachweis für Klusenanlage durch jungtertiäre Flüsse erbracht. Auch der Pontienfluß hatte erst kräftig erodiert, bevor er zur Aufschüttung überging, was sich an der schon J. B. GrepPIN ${ }^{25}$ bekannten Stelle des Plateaus von Sur Chaux bei Courfaivre im Delsbergertal zeigt, wo die Vogesensande seitlich an den Delsbergerkalk angelagert sind; es ist ein fossiles, tertiäres Uferstück. In den Mulden s Courfaivre sind keine pontischen Sedimente bekannt oder erhalten; dagegen öffnen sich, analog wie beim Chaibeux, $s$ des Bois de Raube die Doppelklusen von Undervelier (Fig. 1 und 2). Die Ursorne war also ein aus den Vogesen stammendes Gewässer des Jungtertiärs. Vielleicht haben die Pontienflüsse auch am Chaibeux Spuren hinterlassen, die aber wegen der analogen Sedimente nicht erkannt werden können.

Gestützt auf diese Befunde lassen sich nun vorerst die Klusenverhältnisse im Gesamtjura einer ersten Analyse unterziehen. Überall dort, wo jungtertiäre Schotter aus dem $\mathrm{N}$ bekannt sind, wo sich auf den Ketten auffällige Paßlücken finden (z. B. Pierre Pertuis), wo sich sonderbar verlaufende Flußabschnitte zeigen, wo Klusen auftreten, kann vorerst auf eine vortortonische Anlage geschlossen werden. Eine erste Einschränkung mag bereits hier erwähnt sein. Die Klus bei Court s Moutier hat keine Fortsetzung nach S durch die Montozkette. Es bestand also dort vermutlich noch kein deutliches Gewölbe, in das sich der Fluß bis auf die Juraunterlage hätte einschneiden können. Klusen in den Randketten sind demnach in ihrer Anlage noch näher zu untersuchen. Zum tertiären Flußnetz können z. B. gerechnet werden das Querstück des Doubs s St-Ursanne, alte N-S Rinnen bei Chaux-de-Fonds ${ }^{20}$, die Klusen $s$ des Laufenbeckens bis nach Envelier, diejenigen des Waldenburgertales bis nach Oensingen, ev. auch der Aaredurchbruch n Brugg. Überblickt man die Juranagelfluhkarte von SCHAAD ${ }^{39}$, so fällt die deltaartige Verteilung dieser Kiese auf. SCHAAD fand auf der Lucheren die Juranagelfluh in einer ca. $30 \mathrm{~m}$ tiefen Rinne eingebettet; Vosseler hat auf der Bötzberghochfläche ${ }^{50}$ ein Tälchen von ca. $80 \mathrm{~m}$ Tiefe, von Juranagelfluh zugeschüttet, beobachtet, was auch AmsLer bekannt war ${ }^{2}$. All dies sind Anzeichen für eine vortortonische Erosion durch Flüsse aus dem N, die bis zur Erosionsbasis im Schweizer-Mittelland flossen. Eine weiterausholende Diskussion darüber erfolgt auf S. 296 und S. 301.

\section{Die Verbreitung der Relikte der Höhenschotter.}

Erstmals haben ScHWabe, später RUTTE versucht, die Höhenschotter kartographisch zu erfassen ${ }^{42}{ }^{38}$. Beiläufig sei bemerkt, daß auf Fig. 1 sowohl die Höhenschotter als auch die randlichen Relikte der pontischen Schottervorkommen mit den gleichen Zeichen versehen wurden.

$\mathrm{Da}$ die Höhengerölle als gleichaltrig mit den pontischen Charmoilleschottern aufgefaßt werden, kann man den Charmoillefluß über die Caquerelle, über Bois de Raube, Velleratkette bis nach Tavannes verfolgen, wo jüngere Glazialablagerungen ihn unkenntlich machen, es sei denn, man finde in ungestörter Lagerung typische Vogesenporphyre. Dieser Stromstrich umsäumt auch die Klusen von Undervelier, wo BIRKHÄUSER ${ }^{8}$ auf den Kämmen der Velleratkette Relikte der Quarzitschotter bestätigen konnte; ROTHPLETZ hat typische Vogesengerölle in großer Zahl bis an die Pierre Pertuis gefunden ${ }^{37}$. Die Höhenschotter ergeben ungezwunge: die Fortsetzung der Bois de Raubekiese nach $S$.

ERZINGER ${ }^{20}$ hat in der Ajoie bemerkt, daß sich vereinzelte Vogesengerölle auch außerhalb der Hauptakkumulation verstreut vorfinden, was ja schon vom Bois de Raube bekannt war. Es liegt deshalb die Vermutung nahe, daß die Charmoilleschotter ebenfalls in einer Rinne liegen, die der Fluß zunächst ausfüllen mußte, und die später bei Hochwasser seitlich weit überschwemmt wurde, in viel dünnerer Schicht, so daß. Erzinger eine Strombreite von ca. $20 \mathrm{~km}$ vermutete. Er hat darum den Zusammenhang der Charmoillekiese nach $\mathrm{E}$ mit den Höhenschottern von Scholis, Plainbois und Pleigne postuliert ( siehe Fig. 1) ${ }^{32}$. Diese Angabe über die Streubreite mag sich vielleicht auf $15 \mathrm{~km}$ reduzieren, aber dieser Umstand ist ein wertvoller Hinweis für die Größe der tertiären Stromstriche und vor allem über seitlich anschließende, geröllfreie Zonen. Die Verfolgung ihrer Ausbreitung auf den Jurahöhen bietet eine weitere Methode des Studiums der jungtertiären Gewässer aus dem N.

Östlich Pleigne setzen nämlich die Höhengerölle auf eine Distanz von ca. $10 \mathrm{~km}$ nach $\mathrm{E}$ fast völlig aus; erst $\mathrm{n}$ der Laufenermulde beobachtet man erneut ähnliche 
Formationen, die Wanderblöcke 29. Dieselbe geröllfreie Zone zeigt sich auch im S auch auf den Rücken der Vorburgkette, Velleratkette usw., wo die nächsten Höhengerölle e Undervelier erst bei Moutier, an den Rändern der Birsklusen vorkommen. Dort ist die geröllfreie Zone $5-7 \mathrm{~km}$ breit. Die geröllfreie Zone begleitet also die pontischen Muldenkiese und die Höhenschotter quer durch den Kettenjura hindurch. W des Stromstrichs Charmoille-Bois de Raube sind die Verhältnissc komplizierter; eine eigentliche geröllfreie Zone ist in den Freibergen noch nicht festgestellt. Immerhin haben LAUBSCHER und SchWABE ${ }^{30,}{ }^{42}$ genaue Angaben über ihre Funde auf der Caquerellekette und bei St-Brais gemacht, so daß zu sagen ist, daß das N-S-Querstück des Doubs von Höhenschottern begleitet wird. Der dazugehörende N-S-Fluß hatte wahrscheinlich seinen Ausgang auch in der Caquerellelücke, wäre also mit dem Charmoillestrom identisch und kann als eine zeitlich divergierende Flußrinne in der deltaartigen Aufschüttung des Bois de Raube betrachtet werden.

Die vortortonische Rinne des Chaibeuxflusses mag im Pontien nur schwach benutzt worden sein; Hinweise sind die Vorkommen von Höhenschottern bei Höflein ne Pleigne und diejenigen s von Moutier, zuletzt von $E_{L B E R}$ beschrieben ${ }^{19}$. Östlich des Chaibeuxstromes läßt sich erneut eine geröllarme oder -freie Zone auf den Höhen feststellen, bis man se Laufen auf die Wanderblockformation und auf die Quarzitund Buntsandsteingerölle auf den Ketten um Erschwil, Envelier und Matzendörfler Stierenberg stößt, die die dortigen Klusen umsäumen ${ }^{43}, 19,52$.

Eindeutig scheint auch die Frenkentalrichtung mit den Juranagelfluhaufschüt:tungen $\mathrm{n}$ Waldenburg übereinzustimmen, in deren Stromstrich die Durchbrüche bei Waldenburg, Langenbruck,, Mümliswil und Balsthal liegen. Ihre Ränder schienen von Höhengeröllen frei $z u$ sein, bis überraschend MüHLBERG s Mümliswil ortsfremde Gerölle auf Dogger entdeckte ${ }^{15}$.

Die deutliche und durchgehende Gliederung der allerdings spärlichen Reliktc der Höhenschotter in Geröllzonen und in geröllfreie Zonen von $\mathrm{N}$ nach $\mathrm{S}$ bietet einen weitern Hinweis auf die Gleichaltrigkeit der Bois de Raubekiese und der Höhengerölle. Das jungmiozäne Flußnetz war in unserm Gebiet sehr dicht; es war von der heutigen Tektonik, vom heutigen Faltenverlauf völlig unabhängig, ist also typisch antezedent.

Damit fallen nun aber auch die Gründe dahin, die einige Beobachter bewogen hatten, die Höhengerölle eventuell aus den vicl ältern Nagelfluhen des Helvetien abzuleiten, ganz abgesehen von der von ihnen übersehenen Tatsache, daß die bunte Nagelfluh s der Linie von Moutier aus den Alpen stammt und demnach kein Vogesenmaterial liefern konnte. Natürlich können in Tertiärmulden vereinzelte Gerölle aus der helvetischen Nagelfluh frei geworden sein; sie sind petrographisch von den jüngern, pontischen Kieseln nicht $\mathrm{zu}$ unterscheiden. Aber sie bieten keinen stichhaltigen Grund für Zuweisung der Höhenschotter zum Helvetien und könnten die geröllfreien Zonen in N-S-Richtung nicht erklären.

\section{Erosion und Akkumulution des Jungtertiärs in Verbindung mit den jurassischen Faltungsphasen.}

Burdigalienmolasse ist zur Hauptsache nur im mittelschweizerischen Molassetrog abgelagert worden; ihre N-Grenze verläuft nach BAUMBERGER ${ }^{23}$ vom E-Jura her dem Jurasüdfuß entlang, biegt indessen ungefähr w Solothurn ins Gebiet des Kettenjuras ein, wo sie in der Mulde von Court (und Moutier?) gefunden wird. Diese Einbiegung erfolgt just da, wo die schwarzwäldische Rheintalflexur in ihrer s Fortsetzung ins Mittelland münden würde; sie scheint sich also noch zu Beginn Miozän bemerkbar gemacht zu haben.

In Fig. 3 ist Baumbergers Faziesprofil durch die Raurachische Senke (aus 23) reproduziert; man erkennt darin eine bedeutsame Absenkung am Jurasüdfuß, an der sowohl das mächtige Aqui$\tan$ (Oberoligozän), das im Jura nicht vorkommt, als auch das darüber weggreifende Burdigal transgredieren. Die Flexuranlage erfolgte deutlich voraquitan. Diese S-flexur ist die oben genannte Faziesgrenze des Burdigals in E-Jura; sie fällt zusammen mit dem von BuxroRF im Hauensteinbasistunnel beschriebenen Südtauchen des Tafeljurasüdrandes, ist also Grenze zwischen Molassetrog der Schweiz 
N

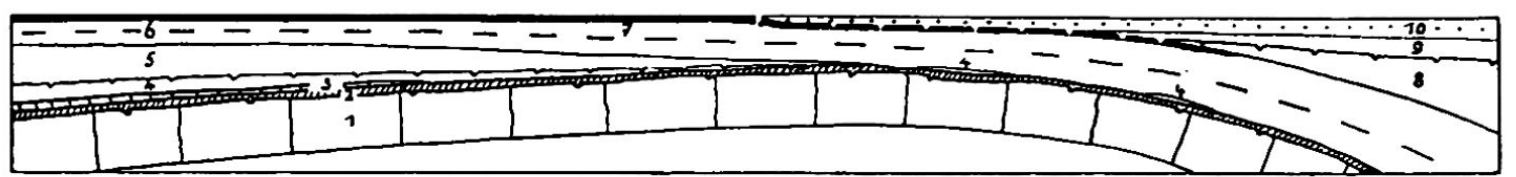

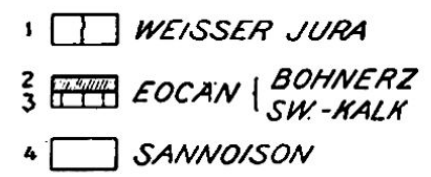

SANNOISON

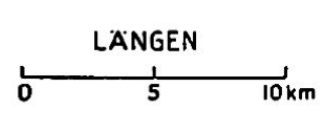

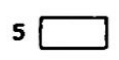

$6--$

7

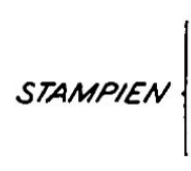

SEPTARIENTON

ELSASSER.

DELÉMONTIEN

TRANSGRESSIONEN

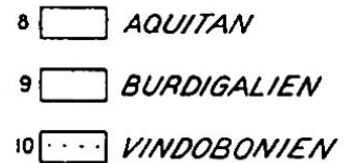

VINDOBONIEN

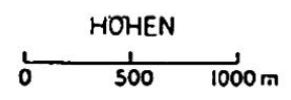

Fig. 3

und Sedimenttafel des Schwarzwaldes. Buxrorf nannte sie die Montterribleflexur; sie ist nicht nur unter dem Hauenstein, sondern nach AMsLeR und HEIM auch unter der Staffelegg und bei Brugg erkennbar als Schwelle im Grundgebirge, „über welche die geschobene Gesteinsmasse des Juragebirges gestrauchelt ist" ( $\left.{ }^{\text {" }}{ }^{2}{ }^{26}\right)$.

BaUmbergers Profil läßt aber noch eine zweite tektonisch wichtige Linie erkennen, die bisher eigentlich unbeachtet blieb. Die S-Flexur ist der Rand eines flachen Rückens aus Jurakalk, der eine Firstlinie bei Delsberg und einen flachen, vorstampisch angelegten Abfall zum Rheintalgraben aufwies. Dieser Monterriblerücken ist wohl nichts anderes als das Gebiet, auf dem später die Jurafaltung erfolgte, also eine Großanlage des Gebirges; seine Firstlinie entwickelte sich zur VorburgRangierskette und weiter im E, wo der Jura schmäler wird, fiel sie örtlich fast zusammen mit der S-Flexur, über welchen Hindernissen sich später die Muschelkalkaufbrüche tïrmten.

Während bis ins Oberstampien die variszischen NNE-SSW-Linien unser Gebiet beherrscht hatten, drang zu Beginn des Aquitans ein neues und wichtiges Element in die Entwicklung des Juras ein: mit der Ausbildung der S-Flexur und des Montterriblerückens begann eine Faltung in W-E, resp. SW-NE-Richtung, die dann im Miozän dominiert. Schon AmsLeR ${ }^{1}$ hatte die Vermutung ausgesprochen, daß die Montterriblelinie mit Faltungsvorgängen zusammenhängen könnte.

Die Ablagerungen des Helvetien wurden bereits in der Einleitung skizziert. Die n Strandzone liegt im Tafeljura auf Malm und Dogger, was auf vorausgegangene Hebung und Abtragung des Gebietes weist; bei Mettemberg $n$ Delsberg ruht die Strandbrekzie ebenfalls auf Malm, was eine erste Anlage der vom Tertiär entblößten Vorburgkette, also eine Verstärkung der Firstlinie des Montterriblerückens bedeutet. Da ferner im E-Jura, im Gebiet der hohen Ketten, Helvetien überhaupt fehlt, haben bereits BUXTORF und nach ihm LEHNER ${ }^{31}$ die Vermutung ausgesprochen, es könnte dort im Helvetien Land, also eine Auffaltung vorhanden gewesen sein. In dieselbe Richtung weist der Umstand, daß im Hauensteinbasistunnel auf der tertiären Transgressionsfläche nur Obermiozän zu konstatieren war $^{12}$.

Für vorhelvetische, schwächere Faltung in den s Teilen plädieren auch die erfolgreichen Untersuchungen von Schlaich ${ }^{41}$ in der Mulde von Court, wo im Burdigalien deutlich S-N laufende Rinnen zu konstatieren sind, aufgefüllt durch die bunte, helvetische Nagelfuh. Neben umgelagertem Burdigal findet man aber auch Gerölle von Delsbergerkalk und von Malm. Auf Grund ähnlicher Befunde konnte RothPLEZ die nachburdigale Faltung bei Tavannes bestätigen ${ }^{3 i}$.

Die bunte helvetische Nagelfluh, die in der $\mathbf{n}$ Litoralzone fehlt, ist möglicherweise eine jüngere Bildung und kann dem mittleren Helvetien zugerechnet werden. Sie enthält Alpengerölle vom Napftypus bis nach Moutier (5) und Vogesenbestandteile bis nach Vermes und Undervelier; die tiefste Mittellandrinne verlief also damals ungefähr in der Zone von Moutier. Diese helvetischen Schotter selbst sind deutliche Anzeichen für das Aufsteigen des Alpenkörpers und der Horste SchwarzwaldVogesen, womit der Zusammenhang zwischen Hebung und Faltungsschub angedeutet ist, während gleichzeitig der mittelschweizerische Trog einsank.

Man kann sich die komplizierten Vorgänge dieser Zeit ungefähr so vorstellen : Nach der Burdigalsenkung und -ablagerung faltete sich der Hauptteil des Montterriblerückens schwach in WSWENE Richtung, sodaß die ersten Kettenanlagen entstanden. Danach trat eine gewisse Ruhe ein, verbunden mit Abtragung bis in den weißen Jura. Es bildete sich die bekannte Vindobon-Peneplain BuXTORFs ${ }^{10}$, ${ }^{81}$, die im Tafeljura durch die Einebnung der Keilgräben besonders deutlich in Erscheinung tritt; diese Einebnung war wohl seit dem Stampien in Funktion. Eine leichte Senkung an der Montterrible-Südflexur ermöglichte dem Helvetienmeer eine kurze Überflutung der Vindobon- 
fläche im Jura, wobei höhere Bezirke frei von Strandbildungen blieben. Die Süßwasserbildungen über dem marinen Helvetien weisen auf sofortige Aussüßung des Meeres und damit auf erneute Hebung, resp. Faltung nach dem Helvetien.

Es sei beiläufig an das bekannte Schema aller Gebirgsbildungen erinnert, das auch hier deutlich vor Augen tritt: Zuerst eine tektonische Phase (verbunden mit Hebungen), dann Abtragung der Formen bis zur Bildung einer ausgeglichenen Fläche, dann Sedimentation und Aufschüttung.

Dieser schematische Ablauf zeigt sich erneut an der Wende des Helvetien zum Torton; die in Abschnitt I beschriebenen Verhältnisse am Hügel Mont Chaibeux machen eine schwache, vortortonische Auffaltung der Velleratkette wahrscheinlich (vortortonische Phase der Faltung). Daraufhin wurde eine Rinne eingetieft, die in die Molasse der Mulde und in den Malm der s folgenden Velleratkette eingriff (Erosionsphase) und danach wurde die Rinne zugeschüttet. Überall im Jura stößt man auf ähnliche Hinweise vortotonischer Faltungstätigkeit. Im Laufenbecken greift Juranagelfluh bis auf Sequan und kappt ältere Gewölbe ${ }^{29}$, im Tonilöchli transgrediert sie auf Sequan ${ }^{52}$, bei Tramelan nach FORKERT ${ }^{21}$ auf Portland, also auf erheblich jüngern weißen Jura. Im Hauensteinbasistunnel sah BuxTORF obermiozäne Konglomerate und Obermiozän auf $20^{\circ}$ s fallenden Effinger-, Geißberger- und Doggerschichten transgredierend, was eine flache, gekappte Falte anzeigt; außerdem ist nach Buxtorf auch die kleine Sprüselfalte außerhalb des Tunnels vortortonisch angelegt worden ${ }^{12}$. Nach VOSSELER ${ }^{\overline{0} 0} 51$. und anderen sind die Faltungen, Überschiebungen usw. der Mettauerund Mandacherzonen vortortonisch oder noch älter.

Intenșität und geographisches Ausmaß dieser zweiten, vortortonischen-Juraphase waren sichtlich bedeutender als in der vorhelvetischen ersten Vorphase. Die Schotterbildungen sind die Folge einer Heraushebung der Vogesen und des Schwarzwaldes; daß nach der Faltung die Erosion, also die Rinnenbildung, auch in den Mulden zu beobachten ist, kann nur dadurch erklärt werden, daß mit den Vogesen auch das ganze Juragebiet sich hob, d.h., vortortonisch erfolgten Faltung und Hebung des Gebietes gleichzeitig.

An der Wende Torton-Pontien, im Sarmatien, wiederholte sich der Ablauf der Ereignisse; erst Hebung und Faltung des Gesamtgebietes, dann Erosion, z. B. kenntlich durch die Ausräumung innerhalb der Rinne des Bois de Raube, hernach Aufschüttung der pontischen Schotter. Diese neue, dritte, vorpontische Faltungsphase ist von ScHWabE in den Freibergen und von den meisten andern Jurabeobachtern erkannt und als die erste Hauptphase der Faltung überhaupt beschrieben worden, allerdings in der Voraussetzung, daß erst mit ihr die eigentliche Aufwölbung eingesetzt habe.

Die große Überschiebung der Trias auf das Tertiär im Basler Jura hat wohl unmittelbar nach dem Obertorton eingesetzt, denn im Hauensteinbasistunnel fehlten jüngere Schichten als Obertorton ${ }^{12}$.

Im Gebiet von Delsberg bestand die Caquerellekluse in der vorhandenen Rangierskette; auf deren Rücken wurden Vogesengerölle auf Dogger abgelagert und Höhenquarzite fanden sich auch auf Dogger $s$ von Delsberg ${ }^{32},{ }^{8}$. Indirekt kann deshalb aus diesen letzteren Tatsachen auch auf eine intensive Erosionstätigkeit nach der zweiten, vortortonischen Faltung geschlossen werden, als vielleicht die Flüsse bis auf das Rauracien abgetragen hatten; die Abtragung im Sarmat hatte dann noch Rauracien und Oxford $\mathbf{z u}$ bewältigen.

Es wird nun immer deutlicher, daß die Gebirgsbildung des Juras nicht im Sinn der frühern Auffassungen verlief, da man meinte, daß eine lange Periode der Sedimentation des Tertiärs am Schluß des Miozäns von zwei kurzen Faltungen beendet wurde, sondern da $\beta$ es sich um eine langsame, fast fortdauernde Hebung und Auffaltung seit der Eozänzeit, gefolgt von Einebnungen, handelt, die durch kürzere Akkumulationsperioden abgelöst worden waren. Die Einebnungen nach der dritten Faltungsphase im Sarmat führten zur letzten Ausgestaltung einer regionalen Peneplain, deren Verfolgung das beste Mittel darstellt, die dritte Faltung und die folgenden Phasen des Jurawerdens zu untersuchen (vergl. Tabelle Seite 298 und 299).

\section{Die sarmatische (sog. vorpontische) Peneplain und die spätern Fultungsphasen.}

Sie ist erkennbar durch die Aufschüttung der pontischen Vogesensande und durch die Relikte der Höhenschotter, die als in situ verarmte, dünne Decke der pontischen Kiese aufgefaßt werden. Der Name "sarmatische "Peneplain stammt von BRAun ${ }^{10}$, der im Tafeljura auf das Bestehen einer jüngern Fläche als die Vindobone Peneplain Buxtorfs hinwies, die weniger steil als jene nach $S$ falle und über dem Dach der Juranagelfluh verlaufe. Vosseler hat sie etwas später im Aargauertafeljura eingehend studiert ${ }^{\mathbf{1 8}}$. Sie ist das genetische und zeitliche Aequivalent der Fläche in der Umgebung von Delsberg, von wo ich sie zuerst bei Pleigne in der Gegend von Movelier beschrieb ${ }^{14},{ }^{32}$. Dort greifen nämlich die Höhenschotterrelikte auf der tischähnlichen Platte über alle Jurastufen des Malms und über eingeebnete Brüche und Überschiebungen hinweg, finden sich dann in der benachbarten Bürkisbergmulde auf Malm und auf dem Doggerrücken der Vorburgkette, so daß ich nicht zögerte, sie mit den Rumpffächen von Caquerelle und der Freiberge zu identifizieren. Natürlich hat diese regionale Fläche auch das Delsbergerbecken überzogen, wo sie über die vermutlich einst von Helvetien bedeckte Tafel des Rückens von Sur Chaux (gebildet von Delsbergerkalk) und über die höchste Tertiärfläche des Mont Chaibeux hinweggriff. Die sarmatische Rumpffäche ist jedoch nicht identisch mit der Auflagerungsfläche der pontischen Bois de Raubeschotter innerhalb der Rinne, welche in die Rumpfebene eingetieft ist. Diese Rinnenfläche ist keine eigene Rumpfebene. Dadurch, daß ich mich darüber nicht genau ausgedrückt und diese Rinne in die sarmatische Fläche mitein- 
bezogen hatte ${ }^{32}$, entstand bei Schivabe, dem Bearbeiter der Morphologie der Freiberge ${ }^{\mathbf{2}}$, die Auffassung, es bestünden zwei Peneplains in den Freibergen, eine ältere als Unterlage der Bois de Raubeschotter, gefolgt von der ersten Hauptfaltung, und eine jüngere, mittelpliozäne, der die Freibergffäche zuzurechnen wäre, gefolgt von der Hauptphase der jurassischen Gebirgsbildung. Dieser Auffassung sind dann später LaUbscher, RUTTE und A.Ninahein gefolgt ${ }^{30},{ }^{42},{ }^{3}$. Durch die in den vorhergehenden Abschnitten erfolgte Präzision ist nun diese Auffassung widerlegt; es existiert im Jura an der Wende des Miozäns zum Unterpliozän nur eine regionale Einebnung und zwar die sarmatische. Sie ist ihrem Wesen nach nur die letzte Ausgestaltung der schon im Burdigal angelegten, südwärts geneigten Abdạchungsfläche von Vogesen und Schwarzwald; auf ihr flossen die pontischen Flüsse nach $S$ bis in die Gegend von Tavannes, wo vielleicht bereits eine Uraare nach $\mathbf{E}$ strömte, und wo die südlichsten Juraketten noch kaum angedeutet waren. Die beute beobachtbaren Verbiegungen und Verstellungen dieser Rumpfebene gaben schon BRÜCKNER und später vielen andern den Hinweis, daß nachpontisch, also etwa im Mittelpliozän, eine erneute Auffaltung einsetzte, die sog. Hauptphase. Es wäre demnach die vierte im miozänen Zyklus. Nach BuxToRF ${ }^{12}$ hat sie die Schenkel der Ketten kräftig steilgestellt, ja zum Teil überkippen lassen, hat die in den frühern Phasen angelegten Überschiebungen vor allem im S-Teil des Gebirges verstärkt, ja sogar verbogen, was im Grenchenbergtunnel schön zu beobachten war. Sie hat die alten, rheintalischen Brüche zum Teil aufgerissen, wodurch scheinbare Querbrüche entstanden. So hat z. B. LAUBscher bei der Caquerelle reaktivierte N-S Brüche entdeckt ${ }^{30}$, was u. a. für die Erklärung der Hochhebung der Freiberge und der Erhaltung der Peneplain von Wichtigkeit ist. Im $S$ des jurassischen Faltenbündels wurden im Gebiet der Südflexur vermutlich schwache Gewölbe in der Molasse zu neuen Ketten emporgequetscht, am S-Schenkel sogar unterschoben und erschienen nun als die höchsten Gewölbescheitel. Die Schubkraft dieser letzten großen Phase erscheint so bedeutend, daß BuxToRf sie mit der spät-insubrischalpinen zeitlich in Beziehung setzte. In dieser Hinsicht sind z. B. die zwei Profile durch Weißenstein- und Chasseralkette in Heims Geologie der Schweiz, Band I, Tafel XXII äußerst treffend. Die südlichste Kette, vorpontisch noch durch keine oder recht schwache Klusenanlagen gekennzeichnet, ist nun nachpontisch die höchste; alle andern nach $\mathrm{N}$ folgenden sind von der nun nach $\mathrm{N}$ geneigten Peneplein gekappt und wenig verändert. SCHWABE hat dabei nicht nur Verstellungen, sondern eigentliche Verbiegungen, z. B. bedeutsam von $W$ nach $E$ (im Caquerellebruchgebiet), um $90-150 \mathrm{~m}$ festgestellt. Betrachtet man die obigen Profile, die durchaus dem Aussehen des ganzen N-Juras im Pontien entsprechen, so ist es verständlich, daß zumindest die heut auf Rauracien oder Dogger aufliegenden Relikte der Höhenschotter nicht sekundär umgelagert sein können. Wenn also RUTTE ${ }^{38}$ diese Quarzitschotter wegen der unrichtigen Deutung eines einzigen Befundes von LAUBSCHER ${ }^{30}$ als völlig unbrauchbar für die Datierung der Faltungsphasen erachtet, so ist dieser Einwand abzulehnen. Natürlich können lokal hie und da Umlagerungen solcher Höhenschotter stattgefunden haben, wie es eben LAUBSCHER oder BITTERLI ${ }^{9}$ beschreiben, aber sie haben mit dem regionalen Gepräge der großen, sarmatischen Peneplain, die in einigen typischen Plateaulandschaften des Gebirges fast unverändert erhalten blieb, nichts zu tun.

Die Tatsache der heutigen, hohen Lage der Freiberge läßt die Frage often, ob noch eine schwächere Nachphase stattgefunden habe, die sich im $\mathbf{N}$ des Gebirges in Hebungen äußerte, vor allem w der Caquerellebruchzone. Man kann diese Hebung aber auch ungezwungen ins Oberpliozän setzen, da ERZINGER in der nördlichsten Ajoie die ins St. Prestien gehörenden Sundgauschotter mit den dortigen, schwachen Antiklinalen gehoben fand ${ }^{20}$. Dadurch kennzeichnet sich eine fünfte tektonische, eine oberpliozäne Nachphase, bei der vermutlich auch kleinere Ketten wie die Bornfalte bei Olten an den Innenrand angegliedert wurden (AMSLER ${ }^{1},{ }^{2}$ ).

Die starke vierte Auffaltung, die nachpontische, hat im Jura eine völlige Revolutionierung des Gewässernetzes bedingt, vor allem, weil nun plötzlich das höchste Gebiet im äußersten S erstand und die Entwässerungsrichtung nach $\mathrm{N}$ verlaufen mußte, anstatt wie im Miozän nach $\mathrm{S}$. Zunächst wurden die Gewässer wohl alle in die neuen Synklinalen abgedrängt, wo sie Auswege suchten und in den schottererfüllten, ältern Klusenrinnen auch fanden, die bald ausgeräumt und vertieft wurden, soda $\beta$ die heutigen Klusen entstanden, die sich mehr und mehr senkrecht zum Streichen der Ketten stellten. Die neue Erosionsbasis bei Basel, geschaffen durch den erst nach $W$ durch die Burgunderpforte fließenden und nachpliozän ins Oberrheintal nach $\mathbf{N}$ strömenden Urrhein, gab diesen Klusenbächen erhöhte Erosionskraft. Die Verteilung der heutigen Klusen ist darum direkt ein Reagens auf die vortortonisch und vorpontisch vorhandenen Falten. Einige der großen Klusen in den Randketten des Gebirges wären demnach erst nachpontisch und diluvial angelegt und durch Rückivärtserosion vertieft. $Z \mathbf{u}$ ihnen gehören wohl die Taubenlochschlucht bei Biel, die Klus durch die Bornfalte und diejenige durch die Landskronkette bei Flüh im Birsigtal, vielleicht auch die Klus von Gänsbrunnen. Erst diese nachpontische Gewässer- und Klusenbildung entspricht dem von A. HeIs gezeichneten Bild der antezedenten Gewässer des Juras. $Z u$ den oben genannten, neu entstandenen Synklinalbächen gehörte vermutlich auch der Urdoubs, der von W gegen St-Ursanne floß und dort irgendwo, vielleicht durch die Caquerellepforte, dem neuen Birslauf oder dem neuen Rhein zufloß. Er hat aber nicht, wie STAUB meinte $\$$, seinen Lauf über die Caquerellekette und über Delsberg genommen. Er muß aber sehr bald von W her rückwärts angezapft worden sein, so da $\beta$ er in das tertiäre Bett des Caquerelle-St-Brais-Flusses geriet, wobei das auffällige Knie s St-Ursanne entstand. Die Darstellung der Gewässerumkehr ist bei SCHwaBE ${ }^{22}$ sehr zutreffend im Detail dargestellt. 
Überblickt man die Übersichtskarten des Juragebirges in der Geologie der Schweiz ${ }^{26}$, Tafel XX oder diejenige von BERsIER ${ }^{23}$, Tafel III, so erfaßt man mit einem Blick die topographische Einheitlichkeit des Ablegers der Alpen, der sich s Genf in einer einzigen Kette vom Stamm löst, bei Vallorbe-Pontarlier sich breit hinlegt und bei Baden wiederum in einer einzigen Falte ausklingt. Das Gebirge ist zwar in der Tat eine topographische Einheit, aber tektonisch und morphologisch aus den verschiedensten Elementen zusammengeschweißt.

Tektonik. In einer ersten, alttertiären, hauptsächlich oligozänen Großphase, die noch zu wenig bekannt ist, wurde unser Gebiet im Vorfeld der Schwarzwald-Vogesenhorste und der Massive der Serre und des französischen Zentralplateaus unter alpinem Druck aus SE in variszischer Richtung schwach NNE-SSW gewellt, verbogen und in Brüche zerteilt ${ }^{32},{ }^{33},{ }^{48}$. Diese alten Strukturen, die nach VonderSCHMITT «en échélon » stehen, verlaufen meist quer zum heutigen Juragebirge, vermutlich bis an die S-Flexur des Montterriblerückens, die sich in der subjurassischen Zone schon im Oberstampien abzuzeichnen begann. Die größten dieser Bruchlinien, vom Rheintalgraben SSW verlaufend, zerlegten das $s$ Vorland in mehrere Schollen mit verschiedener Kleintektonik, die vermutlich im Grundgebirge $\mathrm{N}$-wärts glitten. Vonderschmitr hat die Bedeutung dieser Schollen eingehend diskutiert ${ }^{48}$. Der alpine Druck aus SE, der diese Bruchtektonik schuf, mußte in großen Tiefen wirksam sein; er ist mit der spätern oberfächlichen Abscherung des Sedimentsmantels des Juras nicht identisch.

Voraquitan bis Burdigalien setzte dann die anders geartete, miozäne Großphase der Jurafaltung ein, die die oligozänen Linien beinahe rechtwinklig kreuzte, wenigstens im Berner Jura. Sie begann mit der beinahe W-E laufenden Flexur am Jurasüdfuß und mit der Heraushebung des schwachen Montterriblerückens, dessen Firstlinie die Anlage der sog. Brandungskette Rangiers-Lomont ergab, an die sich nach $\mathrm{S}$ die andern Falten embryonal angliederten. Die gemeinsame Streichrichtung von Flexur und Kettenrichtung deutet darauf hin, daß sie aus gemeinsamer Wurzel stammen; vermutlich sind sie als erste Äußerungen der von BUXToRF erkannten Abscherung des jurassischen Sedimentmantels über den salzführenden Horizonten der Trias aufzufassen, die gemäß den obigen Ausführungen eine rein miozäne Angelegenheit ist. Der alpine Druck aus $\mathrm{S}$, der diese neue Bewegung verursachte, mußte unter andern Bedingungen erfolgt sein als der oligozäne, sonst hätte er bloß eine Verstärkung der variszischen Strukturen bewirkt. So aber erfaßte er den zerbronen Untergrund durch weithinziehende Verbiegungen, die an den Knotenpunkten Axialmaxima oder -minima oder Überschiebungen aufweisen und derart das oligozäne Strukturgewebe noch heut durchschimmern lassen. Dies zeigt sich z. B. sehr deutlich in der hypothetischen Verlängerung der Rheintalflexur von Aesch bis gegen Solothurn oder in der s Fortsetzung der Caquerellebruchzone. Vielleicht streichen diese rheintalischen Brüche auch noch in den Untergrund des mittelschweizerischen 'Troges (früherer Gedanke von C. SCнмIDT), wo sie für die heutige Petrolprospektierung von Bedeutung sein könnten*.

* Diese Auffassung wird bestätigt durch die Festlegung einer 15-20 km breiten "Querzone “ mit Querfaltungserscheinungen im Mittelland bei Freiburg anläßlich von Neuaufnahmen der Molasse für Petroluntersuchungen (Erdölgeolog. Untersuchungen im Schweizer Mittelland zwischen Solothurn und Moudon. Beitr. zur Geologie der Schweiz. Geotechn. Serie, Teil III, 1950). Aber auch im E des Molassetroges scheint der Untergrund in oligozäner Zeit bewegt wordeu zu sein. Die bei Abfassung dieser Schrift durchgeführte Petrolbohrung bei Altishofen (Kt. Luzern) ergab nach Pressemeldungen vom 27. November 1952 ein zu frühes Anfahren der Jurakalkunterlage bei $1303 \mathrm{~m}$ Tiefe statt wie erwartet erst auf ca. $1500 \mathrm{~m}$. Auf dem Jura lag das Aquitan und es fehlte das Mitteloligozän. Man kann daraus auf oberflächlich nicht erkennbare voraquitane Bewegungen (Brüche oder Faltungen mit Diskordanzen) in der Tiefe des Molassetroges schließen. 
Werdegang des Nordschizueizerischen Juragebirges

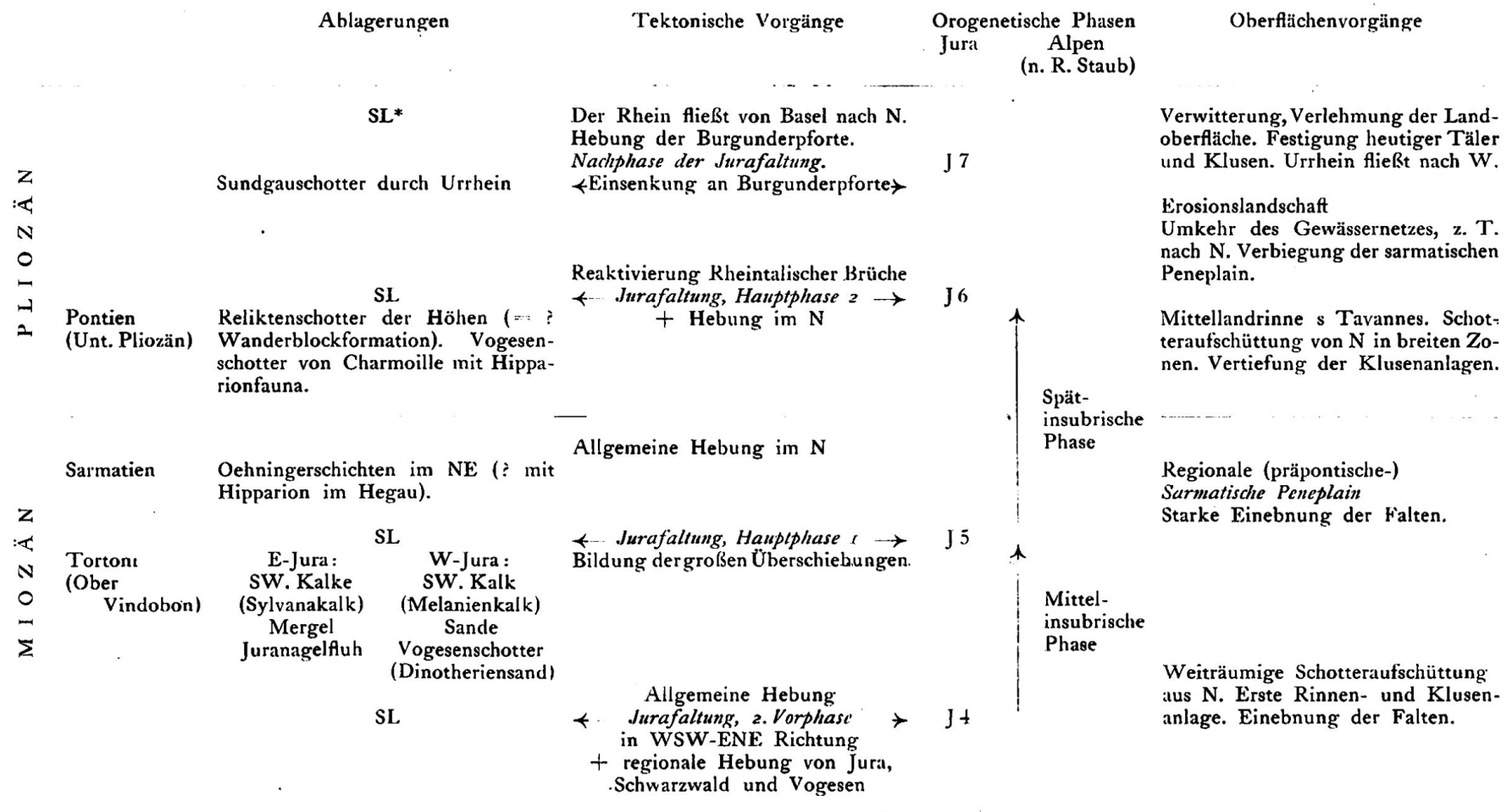




$\begin{array}{ll} & \\ & \\ Z & \\ : 4 \\ N \\ 0 \\ - \\ z\end{array}$

Helvetien SW. Kalke

(Unter Sande

Vindobon) Rote Helizitenmergel

Bunte Nagelfluhdeltas im $S$.

$\begin{array}{cc} & \text { Marines Muschelagglomerat in N } \\ \text { Burdigalien } & \text { Muschelsandstein und Molassegrise }\end{array}$ Muschelsandstein und Mol
Jurasïdfuß und Mittelland

Sannoisien

Bunte Mergel und Mergelsandsteine im Schw. Mittelland

SL

Stampien

Süßwasserkalk (Delsberg u. Tüllingen). Bunte Mergel. Molasse alsacienne, Cyrenenmergel. Meeressand, Septarienton, Konglomerate.

Haustein mit Konglo-

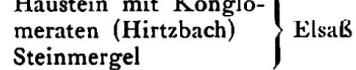

Gelberde, SW. Kalk ... Jura

\begin{tabular}{ll} 
Ludien \\
Bartonien \\
\hline
\end{tabular}

SW. Kalkkonglomerate (Calcaire de Daubrée)

sW. Kalk mit Planorben

Huppersande, Bolus, Bohner

SL Sedimentationslücke $\quad \mathrm{SW} .=$ Süßwasse
Senkungen im Mittelland und Jur: $\leftarrow$ Jurafaltung, $x$. Vorphase $\rightarrow$ in wsw-ene Richtung

Anlage der Brandungskette. Verstärkung prästamp. Querfalten

Hebung von Schwarzwald und Vogesen und Schiefstellung des Vorgeländes

« Verstärkung von MonterribleSüdflexur und Monterriblerücken

[Gropanlage des Inragebirges mit Abscherung]

Senkung im Mittelland

* Anlage Monterrible-Südflexur

und Monterriblerücken in wsw-ene Richtung

Senkungen in Rheintalgraben und "Raurachischer Senke"

Verstärkung der Rheintalbrüche und der Keilgräben

Ferstärkung nne-ssiw Querfalten und von Rheintalbrüchen ? Anlage der Keilgräben (Tafeljura) Senkungen im Rheintalgraben …..........

nne.ssw Querwellen

Rheintalische Brüche (Hirtzbach, Filsa(s)

Embryonale nne-ssw Wellen (Bohnerzzusammenspülung, Delsbg.)
Festland Rasche Aussüßung d. Meeres. Gewässernetz von- $N$ bis

Gewässernetz von S Moutie Einebnung an den Falten.

Transgression des Helvetienmeeres.

insubrische Vindobone Peneplain (Tafeljura). EinPhase ebnungen $s$ von Schwarzwald und Vogesen. Transgression des Burdigalmeeres über Jurasüdfuß.

J2

\section{$\hat{i}$} Monte-

Rosa

Einebnungen

Phase

Tirolide
Phase

Festlandfläche im Jura u. Umgebung Ausfüllung der „Raurachischen Senke“

Arisonide Phase

భ Transgression d. Stampienmeeres $\rightarrow$ auf altstamp. Abrasionsfäclic (Ajoie) Aussüßung. Mariner Vorstoß in den Rheintalgraben. Auffüllungen im Rheintalgraben. Festland im Jura.

A 1, A 2 usw. - alttertiäre, J1, J 2 usw. - jungtertiäre orogenetische Phasen
Festland mit Seen.

(Verwitterungslandschaft) 
Der alpine Druck aus s Richtung hatte drei verschiedene Wirkungen: epirogenetische Hebungen aus sehr großer Tiefe bis weit hinein nach Mitteleuropa, dann Gleitbewegungen des Grundgebirges, die im Altteriär Brüche und Schollen in variszischer Richtung auslösten, endlich die vermutlich aquitan einsetzende Abscherung des Sedimentmantels über 'Trias oder Keuper analog einer alpinen Decke.

Die erste Anlage der Brandungskette vor Burdigal, vorhelvetische Anfügung von Gewölben nach S, auch vortortonisch, das gleichzeitige S-Rücken der tiefsten Rinne des Molassetroges, die Anordnung der Klusenanfänge, die schwache Faltung im N während der vierten Phase und die späte Angliederung der südlichsten Ketten erlauben, den Gedanken BUXTORFs «vom Rückschreitenden Gang der Jurafal. tung $12 \mathrm{zu}$ bejahen und zeitlich festzulegen.

Der nordschweizerische Jura ist ein interessantes Interferenzgebiet zwischen alpiner Faltung und mitteleuropäischer Bruchtektonik. Es wurde darauf verzichtet, die Phasen von Stille zum Vergleich heranzuziehen; dagegen wurden, nach $\mathrm{Ab}$ schì $\beta$ der Arbeit, die von STAUB aufgestellten alpinen Faltungsphasen in die Tabelle eingetragen. Dabei stellte es sich nun heraus, daß die im Jura erarbeiteten orogenetischen und Sedimentationsphasen genau mit den Staubschen aus den Alpen übereinstimmen ${ }^{44}$. Dieses nicht ganz unerwartete Ergebnis ist jedoch deshalb nicht so erstaunlich, weil beide Untersuchungen auf derselben, gegebenen Methode basieren, auf Beobachtung der Diskordanzen und Transgressionen des Tertiärs, vor allem der Schotter, und Rückschlüssen auf die sie verursachenden Bewegungen des Gebirgskörpers. Zudem betreffen sie den gleichen Sedimentationsraum. Voreozäne und diluviale Phasen sind weggelassen. Aber die Übereinstimmung der Resultate dokumentiert doch im großen und ganzen die Richtigkeit der Thesen, zeigt frappierend die Abhängigkeit des Juras von den Alpen, die Gleichzeitigkeit ihrer Vorgänge und erneut die Richtigkeit der Aufïassung, daß das Juragebirge durch alpinen Schub entstand. Es kann nun auch mit Aussicht auf Erfolg der Vergleich der Datierungen in der jurassischen und alpinen Morphologie unternommen werden.

Morphologie. Die Abhandlung hat zu beweisen versucht,, daß die bisherigen Anschauungen über das Werden des Juras insofern revidiert werden müssen, daß die Auffaltung durch das ganze Tertiär hindurch anhielt; längere Perioden von Faltung und nachfolgender Erosion wurden abgelöst von kürzeren Akkumulationszeiten.

Das Tertiär ist erfüllt von Sedimentationslücken. Die Jurafaltung in WSWENE Richtuny war ein seit Beginn Miozän einsetzender, immer stärker in Erscheinung trètender Proze 3 , bei dem die Abtragung bis zur dritten (vorpontischen) Phase der Aufwölbung Meister wurde, während die endogenc Kraft der vierten Phase größer war als die exogene Erosivwirkung.

Die Vergitterung der miozänen Faltung mit den oligozänen Bruchsystemen einerseits und die in den einzelnen Schollen verschieden erfolgte Auffaltung anderseits sind schuld, da3 in diesen Schollen sich verschiedene Stadien der tertiären Landschaftsentwicklung als quasi fossile Landschaften erhalten konnten: Der ElsgauerTafeljura (Ajoie) mit der altstampischen Abrasionsfläche und der östliche Tafeljura mit der Vindobonfläche, dann Laufen- und Delsbergerbecken als Teilstücke des altoligozänen Rheintalgrabens, endlich die Hochflächen von Pleigne-Caquerelle und der Freiberge als Teilstücke der sarmatischen (vorpontischen) Fastebene.

Durch die Ausschaltung der Ansicht, daß das Gebiet der Freiberge einer jüngern Peneplain als der sarmatischen angehöre, ordnet es sich nun in die den ganzen Jura umspannende sarmatische, regionale Peneplain ein. Schon Schwabe hat übrigens mit Recht 12, ${ }^{76}$ die Ebenen von Ornans bei Besançon mit denen des Bötzbergs verglichen. Nach den Beschreibungen von Снавот und Scнwabe ${ }^{18}$, 42 ist kein Zweifel mehr möglich, daß die sarmatische $F$ astebene nach $W$ weit nach Frankreich hinein 
festzustellen ist, wo z. B. bei Ornans nach einer ersten Faltungsphase eine Einebnung erfolgte, die durch eine nachfolgende Faltung verstellt wurde. In der n Ajoic taucht sie unter die Sundgauschotter cin und in den hohen Ketten s des Gebiets von Delsberg ist sie nur noch auf den Faltenrücken, z. T. auch an den gekappten Faltenschenkeln erkennbar. In den Becken von Laufen und Delsberg liegt die sarmatischc Peneplain über der Juranagelfluh und kappt die benachbarten Ketten; in den s Mulden dagegen kann sic wegen der starken diluvialen Ausräumung nirgends mehr mit Sicherheit gefunden werden. Östlich der Rheintalflexur gehören das hochgelegene Gempenplateau (Fig. 1) und die Flächen des Bötzberges und des Thiersteinerberges dazu, wo Vosseler die Peneplain, die über der Juranagelfluh liegt, von Härtlingen überragt fand, was auch bei Ornans der Fall ist. Schon Buxtorf und $\mathrm{KocH}$ wiesen auf die Wahrscheinlichkeit der Fortsetzung der sarmatischen Peneplain im Klettgau und im schwäbischen Jura hin, wo sich hochgelegene Quarzitschotter und Hipparion führende Ablagerungen finden. Ähnliche Vorkommen zeigen sich auch weiter $\mathrm{e}$ in der bayrischen und österreichischen Molassezone und vermitteln den Anschluß an die fluviatilen Hipparionschichten der Belvedereschotter im Wienerbecken. Nördlich unseres Gebietes dürfte die regionale Peneplain an den $S$-hängen von Schwarzwald und Vogesen in Resten auffindbar sein und sich an den Außenhängen der Gebirge nach $N$ verfolgen lassen. Wenigstens hat BodeN in Lothringen bei Blâmont ${ }^{11}$ pliozäne Sande auf einer "greisenhaften 》 Fastebene vorgefunden, und im Mainzerbecken liegen nach $W_{E \times z}$ die Dinotheriensande und Prososthenienschichten diskordant über dem ältern Tertiär und sind zu verknüpfen mit den bekannten Kieseloolithschottern auf dem Rheinischen Schiefergebirge. Durch diese weit ausgreifender Hinweise ist die regionale Bedeutung dieser vorpontischen Einebnung klargestellt.

Es bleibt noch übrig, ihre Zusammenhänge nit den präglazialen Einebnungen in den Alpen zu suchen. Vielversprechende Anfänge sind bereits von BECK, ANNAHEIM ${ }^{7},{ }^{3}$ und anderen gemacht. Ersterer hat im Berner Oberland auf das Simmenfluhniveau als wahrscheinliches Aequivalent hingewiesen, letzterer hat das Pettanettosystem des 'Tessins in diese Fläche einbezogen und außerdem den Versuch gemacht, die sarmatischen Flächen von Ost- und Westalpen mit denen des Juras zu vergleichen.

Die vorliegende Arbeit hat auch einen Beitrag zur Klassifikation der Juraklusen geliefert. Man kann drei Haupttypen von Juraquertälern unterscheiden:

a) Die Klusen des Neuenburger- und Waadtländerjuras, die an den großen Querbrüchen entstanden sind. Sie sind vermutlich tektonisch bedingt.

b) Klusen, die in den Rinnen cines helvetisch-vorportonischen Gewässernetzes in N-S-Richtung sich während den Juraautïaltungen bildeten und die ältesten Quertäler des Gebirges darstellen. Sie zeigen sehr ausgeglichene morphologische Verhältnisse. Nach ihrem cpigenetischen Einschneiden wechselte in ihnen nach dem Mittelpliozän die Entwässerungsrichtung.

c) Klusen, die erst nach der zweiten Hauptphase der Jurafaltung entstanden und noch heute unausgeglichene Verhältnisse aufweisen. Sie sind meist durch rein lokale Erosionswirkungen zu erklären und zeigen sich vornehmlich an den Rändern des Gebirgssystems.

Zum Schluß sei noch eine wichtige Schlußfolgerung aus den tektonischen und morphologischen Betrachtungen erwähnt: der Faktor Zeit ist bei allen Entwicklungen viel mehr in Betracht zu ziehen.

Die Vindobonfläche konnte sich vom Stampien bis Beginn des Helvetien, dic sarmatische Peneplain vom Burdigalien bis Beginn Pontien langsam herausbilden; die meisten großen Klusen des Gebiets sind nicht erst seit dem Diluvium, sondern schon in den bedeutenden Zeiträumen des obern Miozäns angelegt worden und die 
Juraketten selbst sind gemäß den vertretenen Auffassungen nicht das Werk einer kurzen Epoche, sondern wie die Alpen in fast ununterbrochenem Stau im Miozän und Pliozän aufgewölbt worden. Damit scheint mir der zeitliche Ablauf der Ereignisse dẹr Sphäre der Wahrscheinlichkeit viel näher gerückt zu sein.

* Abkürzungen in Text und Tabelle.

Hochstehende Zahlen heziehen sich auf die Nummer im Literaturrerzeichnis. $\mathrm{N}=$ Nord, $\mathrm{E}=$ Ost usw.; $\mathrm{n}=$ nördlich, $\mathrm{e}=$ östlich usw.

\section{LITERATURVERZEICHNIS}

1. ANsLER, A.: Tektonik des Staffeleggebiets. Ecl. geol. H. XIII, 1915. 2. AMsıl.E, A.: Beziehung zwischen Tektonik und tertiärer Hydrographie im östl. Jura. Ecl. geol. H. XVI, 1922. 3. ANNAHEIM, H.: Chronological Correlation of the Morphologic Development in the Eastern and Western Alps. Experientia, Basel. VI, 195, 1950. 4. Baumberger, E.: Bohnerz in „Die Eisen- und Manganerze der Schweiz“, Kümmerly \& Frey, 1 23 . 5. Baumberger, E.: Die Transgression des Vindobonien in den Tertiärmulden von Moutier und Balsthal. Ecl. geol. H. XVII, 1923. 6. BauNBerger, E.: Die stampischen Bildungen der Nordwestschweiz und ihrer Nachbargebiete. Ecl. geol. H. XX, 1927. 7. BECK, P.: Über das schweizerische und europäische Pliozän und Pleistozän. Ecl. geol. H. XXVI, 1933. BIRKIÏUSER, M.: Geologie des Kettenjura bei Undervelier. Verhdl. Natf. G. Basel, XXXVI, 1924/25. 9. Brtrerli, P.: Geologie der Blauen- und Landskronkette s von Basel. Beitrag zur geol. Karte der Schweiz. NF 81, 1945. 10. Braun, G.: Zur Morphologie der Umgebung von Basel. Verhdl. Natf. Ges. Basel, XXVIII, 1917. 11. BoDEN, K.: Die pliozänen Ablagerungen im Gebiet des Oberlaufs der Vezouse in Lothringen. Sitzungsbericht Bayr. Akad. d. W. 1919. 12. Buxtorf, A.: Prognosen und Befunde b. Hauensteinbasis- und Grenchenbergtunnel und der Bedeutung der letztern für die Geologie des Juragebirges. Verhdl. Natt. G. Basel, XXVII, 1916. 13. BuxTORF, A.: Zur Altersfrage der Faltungsphasen im Kettenjura. Ecl. g. H. 31, 1938. 14. BuxtoRf, A. und Косн, R.: Zur Frage der Pliozänbildungen im Nordschweizerischen Juragebirge. Verhdl. Natf. Ges. Basel. XXXI, 1920. 15. Buxtorf, A. und Christ, P.: Erläuterungen zu Blatt Laufen-Mümliswil des Geol. Atlas der Schweiz 1:25000, 1936. 16. Carte géologique de la France 1:50000, Ferrette, 1927. 17. Davies, WM. und Braun, G.: Grundzüge der Physiogeographie, Leipzig und Berlin, 1915. 18. Chabot, G.: Les Plateaux du Jura Central. Etude de Morphogénie. Publ. de la fasc. d. lettres de l'univ. de Strasbourg. 41, 1927. 19. ElBer, R.: Geologie der Raimeux- und der Velleratketten im Gebiet der Durchbruchstäler von Birs und Gabiare. Verh. Natf. Ges. Basel, XXXII, 1920/21. 20. ERZinger, E.: Die Oberflächenformen der Ajoie (Berner Jura). Mittlg. der Geogr. Ethnol. Ges. Basel, Bd. VI, 1943. 21. Forkert, E.: Geolog. Beschreibung des Kartengebietes Tramelan im Berner-Jura. Ecl. geol. H. XXVL, 1933. 22. GlausER, A.: Geolog. Beschreibung des Kartengebiets. Bl. Montfaucon im Berner-Jura. Verhdl. Natf. Ges. Basel, XLVII, 1936. 23. Geolog. Führer der Schweiz. Geolog. Kommission, Basel, 1934. 24. Grahmans, R.: Der Jura der Pfirt im Oberelsaß. N. Jahrb. f. Min. usiv. Beilageband XLIV, 1920. 25. Greppin, J. B.: Description géol. du Jura Bernois. Mat. p. l. Carte Géol. de la Suisse. 8. livre, 1870. 26. Heim, Alb.: Geologie der Schweiz, I und II, 1920. 27. Hummel, K. L.: Die Tektonik des Elsgaus. Ber. Natf. Ges. Freiburg i. Br., XX, 1914. 28. Kel..ER, W.T.: Geol. Beschr. des Kettenjuras zwischen Delsbergerbecken und Oberrheinische Tiefebene. Ecl. geol. H. XVII, 1922. 29. KocII, R.: Geol. Beschr. des Beckens von Laufen im Berner-Jura. Beitrag zur geol. der Schweiz, NF. 48, 1923. 30. Laubscher, H.: Geologie des Gebietes vom Siegfried-Blatt St-Ursanne (Berner-Jura). Beitr. z. geol. K. d. Schw. NF. 92, 1948. 31. LeHNER, E.: Geologie d. Umgebung von Bretzwil i. N. schweiz. Juragebirge. Beitr. z. geol. K. d. Schw. NF. XLVII, 1920. 32. LinIGER, H.: Geologie des Delsbergerbeckens u. d. Umgebung von Movelier, Beitr. ¥. geol. K. d. Schw. NF. 55, 1925. 33. LiNiger, H : Die Gitterfaltung im Berner-Jura. Ecl. geol. H. XIX, 1926. 34. LINIGER, H.: Beitrag zum Klusenproblem. Leben und Umwelt, Basel, 8. Jahrgang, 1952. 35. PHiLıpp, H.: Saône-Rhône-Senke und Jura in ihren Beziehungen zur saxorischen Tektonik. Geol. Rundschau, XXVI, 1935. 36. Rolr..er, L.: Deuxième supplément à la description géol. de la partie de la feuille VII, 1:100000. Mat.p.la carte géol. de la Suisse NF. 8. livre. 37. RothPLETZ, W.: Geol. Beschr. der Umgebung von Tavannes. Verhdl. Natf. Ges. Basel, XVII, 1932. 38. Rutre, E.: Jungtertiär und Altdiluvium im s Oberrheingebiet, Bericht d. Natf. Ges. zu Freiburg i. Br. Bd. 40, 1950. 39. SchaAd, E.: Die Juranagelfluh. Beitr. z. geol. K. d. Schw. NF. 22, 1908. 40. SchafFer, F. X.: Geologie von Oesterreich, Wien 1951. 41. Schlaich, E.: Geol. Beschr. von Court im Berner Jura mit besonderer Berücksichtigung der Molassebildungen. Beitr. z. geol. K. d. Schw. NF. 26, 1934. 42. SchwaBE, E.: Morphologie der Freiberge (Berner Jura). Mittlg. der Geogr.-Ethnogr. Ges. Basel, Bd.V, 1935-38. 43. STähtLin, P.: Geologie der Juraketten bei Welschenrohr (Kt. Solothurn). Beitr. z. geol. K. d. Schw. NF. 55, 1924. 44. STAUB, R.: Grundzüge und Probleme alpiner Morphologie. Denkschrift schw. Natf. Ges. Bd. LXIX, 1934. 45. ToBiEs, H.: Über Hipparionreste in obermiozäner Süßwassermolasse Südwestdeutschlands. Deutsche Geol. Ges. Bd 90, 1938. 46. Virieux, A.: Contribution à l'étude de la genèse des cluses. Actes de la Société Jurassienne d'Emulation. Porrentruy, 1950. 47. Vocel, H.: Geologie des Graitery und des Grenchen- 
bergs in Juragebirge. Beitr. z. geol. K. d. Schw. NF. 26. 1934. 48. Vonderschmir, L.: Die geolog. Ergebnisse der Bohrungen von Hirtzbach bei Altkirch (Ob. Elsaß). Ecl. geol. H. XXXV. 1942.49. Vosseler, P.: Morphologie des Aargauer Tafeljuras (Verhlg. Natf. Ges. Basel, XXIV, 1918. 50. VosSEler, P.: Die tertiäre Entwicklung des Aargauer Tafeljura. Mittlg. Aarg. Natf. Ges. 17, 1926. 51. Vosseler, P.: Die Altformen des Juras, ihre Entwicklung und Verbreitung. Verhdl. d. Schw. Natf. Ges. 1940. 52. WAIBEL, A.: Geologie der Umgebung von Erschwil. Beitr. 2. geol. K. d. Schw. NF. 55, 1925. 53. Wenz, W.: Das Mainzerbecken und seine Randgebiete, Heidelberg $1921,54$. Witrmani, O.: Geolog. Spezialkarte von Baden 1:25000, Erläuterungen Blatt Lörrach u. Bl. Weil, 1952. 55. Wyss, Fr.: Zum Begriff „Klus“ u. s. Anwendung in der Kartographie. Der Schweizer Geograph, Jahrgang 10, 1933.

\section{A PROPOS DE L'HISTOIRE}

\section{E'C DE LA MORPHOLOGIE DES CHAÎNES SEPTENTRIONALES DU JURA}

L'auteur distingue dans la première période du Tertiaire 3 phases orogéniques divisant la région en parties orientées du NNE au SSO. Plus tard, ces parties furent reprises par le plissement, mais d'une façon différente. Les plissements aquitaniens et miocènes ont été classés en 5 ou 7 phases distinctes: 2 plissements faibles à l'Aquitanien, un plissement faible précédant l'Helvétien, un autre, un peu plus accentué, avant le Tortonien, 2 plissements considérables, l'un avant le Sarmatien, l'autre après le Pontien, et enfin un plissement postérieur coïncidant avec le Pliocène supérieur. Le Jura ne doit donc pas sa formation ì deux phases orogéniques du Miocène supérieur; il est le résultat d'une suite de plissements qui ont eu lieu entre le début de l'Eocène et le début du Diluvium. Les phases orogéniques du Jura correspondent à celles qui ont été reconnues par R. STAUB dans les Alpes. Les chaines septentrionales du Jura sont les plus anciennes (conformément aux résultats des recherches de A. Buxrorf). Arant la sédimentation des cailloutis pontiens à Hipparion de Charmoille (Ajoie) une pénéplaine régionale sarmatienne s'était formée, s'étendant de la Franche-Comté jusqu'à l'Allemagne et aux Alpes. L'opinion de R. SCHWabE selon laquelle les Franches-Montagnes formeraient une seconde pénéplaine est donc réfutée. Les nombreuses cluses (celle de la Birse, p.ex) sont d'origine anté-tortonienne et servirent de débouché à un réseau fluvial dont les eaux se sont mises à couler en sens inverse au Pliocène moyen. (Antécédence).

\section{A PROPOSITO DELLA GEOMORFOLOGIA DEL GIURA SVIZZERO SETTENTRIONALl:}

L'autore distingue nel primo periodo terziario 3 fasi orogeniche che hanno diviso la regione in parti orientate da NNE a SSO (che più tardi furono ripiegate in modo diverso) e $5-7$ fasi nel periodo aquitaniano e miocenico: due deboli fasi nellaquitaniano, una debole fase ante-elveziana, un'altra, alquanto più forte, antetortoniana, due fasi considerevoli ante-sarmatiane e postpontiane (fasi principali) e una fase posteriore nel pliocene superiore. Il corrugamento del Giura sarebbe dunque un lungo processo inizitosi fin dal principio dell'Eocene e terminato all'inizio del Diluviale, e non un ripiegamento in due fasi durante il Miocene superiore. Le fasi orogenetiche del Giura corrispondono a quelle riconosciute da R. STAUB per le Alpi. Le catene settentrionali sono le più antiche (conferma dei risultati delle ricerche di A. BuxTORF). Prima della sedimentazione delle arenarie pontiane a Hipparion di Charmoille (Ajoie) si formò un penepiano sarmatiano regionale che dalla Franche-Comté si alla estendeva fino Germania e anche nelle Alpi. Vien confutata l'opinione di R. SCHI'ABE, che le Franches-Montagnes formino un secondo penepiano più recente. Numerose chiuse (cluses) (per es. le chiuse della Birse) sono di origine ante- tortoniana e servirono da scolatoi a una rete fluviale che, nel medio-pliocene, si mise a scorrere in senso opposto (antecedenza).

\section{DAS FISCHEREIGEBIET DER GROSSEN SEEN NORDAMERIKAS}

Fritz BarT7.

Mit 2 Abbildungen

Die 6 großen Seen Nordamerikas bilden mit $250000 \mathrm{~km}{ }^{2}$ Oberfläche das ausgedehnteste zusammenhängende Binnenwassergebiet der Welt. Sie liegen im Übergangsbereich zwischen den gemäßigten und den borealen Klimaten. Die laubabwerfenden Hartholzwälder des Ostens reichen noch an die unteren Seen heran, während die Gestade der Nordküsten der drei größten Seen von subpolarer 'Taiga eingerahmt 